\title{
ON RAMANUJAN SUMS OF A REAL VARIABLE AND A NEW RAMANUJAN EXPANSION FOR THE DIVISOR FUNCTION
}

\author{
MATTHEW S. FOX AND CHAITANYA KARAMCHEDU
}

\begin{abstract}
We show that the absolute convergence of a Ramanujan expansion does not guarantee the convergence of its real variable generalization, which is obtained by replacing the integer argument in the Ramanujan sums with a real number. We also construct a new Ramanujan expansion for the divisor function. While our expansion is amenable to a continuous and absolutely convergent real variable generalization, it only interpolates the divisor function locally on $\mathbb{R}$.
\end{abstract}

\section{INTRODUCTION}

In [5], Ramanujan introduced the exponential sums $c_{q}: \mathbb{N} \rightarrow \mathbb{C}$ defined by

$$
c_{q}(n):=\sum_{k \in(\mathbb{Z} / q \mathbb{Z})^{\times}} e^{2 \pi i k n / q}
$$

for any two natural numbers $q$ and $n$. These sums are known as Ramanujan sums and have many remarkable properties. For example, $c_{q}(n)$ is integer-valued, $q$-even in the argument $n$, and multiplicative in the index $q$. See the surveys [3, 4] and books [6, 7] for details and more. Additionally, several important arithmetic functions can be expressed as a linear combination of Ramanujan sums of the form

$$
f(n)=\sum_{q \geq 1} \widehat{f}(q) c_{q}(n)
$$

for appropriate complex numbers $\widehat{f}(q)$. If this series converges, we say the arithmetic function $f(n)$ admits a Ramanujan expansion. One famous example is the divisor function $\sigma_{k}(n):=$ $\sum_{d \mid n} d^{k}$, which admits the Ramanujan expansion

$$
\sigma_{k}(n)=n^{k} \zeta(k+1) \sum_{q \geq 1} \frac{c_{q}(n)}{q^{k+1}} .
$$

This series converges absolutely when $k>0$ since $\left|c_{q}(n)\right| \leq \sigma_{1}(n)$ for every $q[2]$. Note, however, that this and all other Ramanujan expansions are never unique since $\sum_{q \geq 1} c_{q}(n) / q=0$ for all $n$ [5].

Several studies have generalized the notion of Ramanujan expansions to a wider class of functions. For example, Cohen has formulated a theory of Ramanujan expansions for unitary analogues of arithmetic functions [1, while Tóth and Ushiroya have developed a generalization to multivariate arithmetic functions [8, 9. However, these generalizations all remain within the context of arithmetic functions. It seems interesting, therefore, to formulate a theory of Ramanujan expansions for non-arithmetic functions, like functions of a real variable. In the closing to [4, Murty suggests a simple way one might do this: "The interesting thing about the right hand side of $[(2)]$ is that if the series converges absolutely and we replace $n$ by a real number $x$, we obtain a continuous function which interpolates the given arithmetical function. In this way, we can view the Ramanujan expansion as a continuous analogue of the discretely defined arithmetical function." 
In this note, we briefly explore Murty's idea of generalizing (11) to the continuous sum $\widetilde{c}_{q}$ : $\mathbb{R} \rightarrow \mathbb{C}$ defined by

$$
\widetilde{c}_{q}(x):=\sum_{k \in(\mathbb{Z} / q \mathbb{Z})^{\times}} e^{2 \pi i k x / q}
$$

for any real number $x$ and natural number $q$. Since $c_{q}(n)=\widetilde{c}_{q}(n)$ for all natural numbers $n$ and $q$, the function

$$
\widetilde{f}(x):=\sum_{q \geq 1} \widehat{f}(q) \widetilde{c}_{q}(x)
$$

will indeed interpolate the arithmetic function $f(n)$ in (2), provided (5) converges. The convergence properties of (5) are thus central to the viability of Murty's idea. In this paper we explore what, if anything, the absolute convergence of (2) implies about the limiting behavior of (5).

Heuristically, one might expect $\widetilde{c}_{q}(x)$ to behave roughly like a sum of $\left|(\mathbb{Z} / q \mathbb{Z})^{\times}\right|=\varphi(q)$ random complex numbers, where the real and imaginary parts are each uniformly distributed on the interval $[-1,1] \subset \mathbb{R}$. Insofar as this approximation holds, one would expect $\widetilde{c}_{q}(x)=\mathcal{O}_{x}(1)$ 1 since the expectation value of the sum equals zero. Indeed, this behavior is also what one would naturally intuit from the asymptotic behavior of $c_{q}(n)$. In this context, therefore, it appears reasonable to adopt Murty's expectation that the absolute convergence of (2) implies the absolute convergence of (5).

Surprisingly, however, this intuition is flawed due to the following theorem (Section 2):

Theorem 1. For fixed $x \in \mathbb{R} \backslash \mathbb{Z}$ and all $q>2 \pi|x|$,

$$
\widetilde{c}_{q}(x)=\frac{e^{2 \pi i x}-1}{2 \pi i x} \varphi(q)+\mathcal{O}_{x}\left(\sigma_{0}(q)\right) .
$$

In other words, $\widetilde{c}_{q}(x)=\mathcal{O}_{x}(\varphi(q))$ for all $q>2 \pi|x|$. In big- $\Omega$ notation, it follows that the real variable generalization $\widetilde{f}(x)$ of the arithmetic function $f(n)$ will diverge for all $x \in \mathbb{R} \backslash \mathbb{Z}$ whenever the Ramanujan coefficients $\widehat{f}(q)$ of $f(n)$ are $\Omega\left(q^{-2}\right)$. As an example, we show that although the Ramanujan expansion (3) for the sum-of-divisors function, $\sigma_{1}(n)$, converges absolutely, its real variable generalization does not:

Corollary 1. The generalized sum-of-divisors function,

$$
\widetilde{\sigma}_{1}(x):=x \zeta(2) \sum_{q \geq 1} \frac{\widetilde{c}_{q}(x)}{q^{2}},
$$

diverges for all $x \in \mathbb{R} \backslash \mathbb{Z}$.

This raises the question, does there exist a continuous and absolutely convergent Ramanujan expansion that interpolates $\sigma_{1}(n)$ ? In Section 3, we establish that there does, but it is unnatural because it only interpolates $\sigma_{1}(n)$ in the open neighborhood $(n-1, n+1) \subset \mathbb{R}$. Consequently, our work leaves open the question of whether there exists a continuous and absolutely convergent Ramanujan expansion that interpolates $\sigma_{1}(n)$ globally on $\mathbb{R}$, or at least on the positive reals.

\section{Proofs of Results}

The proof of Theorem 1 relies on the following lemma:

Lemma 1. If $x \in \mathbb{R} \backslash \mathbb{Z}$ and $d>2 \pi|x|$, then

$$
\frac{1}{e^{-2 \pi i x / d}-1}=-\frac{d}{2 \pi i x}+\mathcal{O}_{x}(1)
$$

\footnotetext{
${ }^{1}$ We use the asymptotic notation $f=\mathcal{O}_{x}(g)$ to denote the estimate $|f| \leq C_{x} g$ for some absolute constant $C_{x}>0$ that may depend on the parameter $x$.
} 
Proof. The result follows immediately from Taylor-expanding the exponential.

Theorem 1. For fixed $x \in \mathbb{R} \backslash \mathbb{Z}$ and all $q>2 \pi|x|$,

$$
\widetilde{c}_{q}(x)=\frac{e^{2 \pi i x}-1}{2 \pi i x} \varphi(q)+\mathcal{O}_{x}\left(\sigma_{0}(q)\right) .
$$

Proof. The sum of the Möbius function over the divisors of $n, \sum_{d \mid n} \mu(d)$, is one if $n=1$ and zero otherwise. Hence, $\widetilde{c}_{q}(x)$ in (4) is the same as

$$
\widetilde{c}_{q}(x)=\sum_{k=1}^{q} e^{2 \pi i k x / q} \sum_{d \mid(k, q)} \mu(d)=\sum_{d \mid q} \mu(d) \sum_{\substack{k=1 \\ d \mid k}}^{q} e^{2 \pi i k x / q}=\sum_{d \mid q} \mu(d) \sum_{l=1}^{q / d} e^{2 \pi i l d x / q} .
$$

Since $x \in \mathbb{R} \backslash \mathbb{Z},\left|e^{2 \pi i l d x / q}\right|<1$, so we can apply the geometric series formula to the inner sum:

$$
\widetilde{c}_{q}(x)=\left(1-e^{2 \pi i x}\right) \sum_{d \mid q} \frac{\mu(d)}{e^{-2 \pi i d x / q}-1}=\left(1-e^{2 \pi i x}\right) \sum_{d \mid q} \frac{\mu(q / d)}{e^{-2 \pi i x / d}-1} .
$$

Since $q>2 \pi|x|$, we can partition this sum into a sum over $d<2 \pi|x|$ and another over $d>2 \pi|x|$,

$$
\widetilde{c}_{q}(x)=\left(1-e^{2 \pi i x}\right) \sum_{\substack{d|q \\ d<2 \pi| x \mid}} \frac{\mu(q / d)}{e^{-2 \pi i x / d}-1}+\left(1-e^{2 \pi i x}\right) \sum_{\substack{d|q \\ d>2 \pi| x \mid}} \frac{\mu(q / d)}{e^{-2 \pi i x / d}-1}
$$

Lemma 1 allows us to write the $d>2 \pi|x|$ sum as

$$
\sum_{\substack{d|q \\ d>2 \pi| x \mid}} \frac{\mu(q / d)}{e^{-2 \pi i x / d}-1}=-\frac{1}{2 \pi i x} \sum_{\substack{d|q \\ d>2 \pi| x \mid}} \mu\left(\frac{q}{d}\right) d+\sum_{\substack{d|q \\ d>2 \pi| x \mid}} \mu\left(\frac{q}{d}\right) \mathcal{O}_{x}(1) .
$$

Since

$$
\varphi(q)=\sum_{d \mid q} \mu\left(\frac{q}{d}\right) d=\sum_{\substack{d|q \\ d<2 \pi| x \mid}} \mu\left(\frac{q}{d}\right) d+\sum_{\substack{d|q \\ d>2 \pi| x \mid}} \mu\left(\frac{q}{d}\right) d
$$

and, similarly,

$$
\mathcal{O}_{x}\left(\sigma_{0}(q)\right)=\sum_{d \mid q} \mu\left(\frac{q}{d}\right) \mathcal{O}_{x}(1)=\sum_{\substack{d|q \\ d<2 \pi| x \mid}} \mu\left(\frac{q}{d}\right) \mathcal{O}_{x}(1)+\sum_{\substack{d|q \\ d>2 \pi| x \mid}} \mu\left(\frac{q}{d}\right) \mathcal{O}_{x}(1)
$$

it follows that

$$
\sum_{\substack{d|q \\ d>2 \pi| x \mid}} \frac{\mu(q / d)}{e^{-2 \pi i x / d}-1}=-\frac{1}{2 \pi i x} \varphi(q)+\mathcal{O}_{x}\left(\sigma_{0}(q)\right)-\frac{1}{2 \pi i x} \sum_{\substack{d|q \\ d<2 \pi| x \mid}} \mu\left(\frac{q}{d}\right) d-\sum_{\substack{d|q \\ d<2 \pi| x \mid}} \mu\left(\frac{q}{d}\right) \mathcal{O}_{x}(1) .
$$

Now consider the $d<2 \pi|x|$ sum in (7). Since $q$ has at most $\lfloor 2 \pi|x|\rfloor$ divisors less than $2 \pi|x|$,

$$
\left|\sum_{\substack{d|q \\ d<2 \pi| x \mid}} \frac{\mu(q / d)}{e^{-2 \pi i x / d}-1}\right| \leq \sum_{j=1}^{\lfloor 2 \pi|x|\rfloor}\left|\frac{1}{e^{-2 \pi i x / j}-1}\right|=\mathcal{O}_{x}(1) .
$$

By a similar argument, the two $d<2 \pi|x|$ sums in (8) are also $\mathcal{O}_{x}(1)$. Thus, together (7) and (8) imply

which completes the proof.

$$
\widetilde{c}_{q}(x)=\frac{e^{2 \pi i x}-1}{2 \pi i x} \varphi(q)+\mathcal{O}_{x}\left(\sigma_{0}(q)\right)+\mathcal{O}_{x}(1),
$$


This theorem implies the following generalization of Corollary 1:

Corollary 2. The generalized divisor function

$$
\widetilde{\sigma}_{k}(x):=x^{k} \zeta(k+1) \sum_{q \geq 1} \frac{\widetilde{c}_{q}(x)}{q^{k+1}}
$$

converges absolutely for all $x \in \mathbb{Z}$ if $k>0$ and for all $x \in \mathbb{R}$ if $k>1$, but diverges for all $x \in \mathbb{R} \backslash \mathbb{Z}$ if $k=1$.

Proof. If $x \in \mathbb{Z}$, then $\widetilde{\sigma}_{k}(x)=\sigma_{k}(x)$, which converges absolutely when $k>0$ by the argument after (3). If $x \in \mathbb{R}$, then for $k>1$,

$$
\sum_{q \geq 1}\left|\frac{\widetilde{c}_{q}(x)}{q^{k+1}}\right| \leq \sum_{q \geq 1} \frac{\varphi(q)}{q^{k+1}}=\frac{\zeta(k)}{\zeta(k+1)}<\infty .
$$

If $x \in \mathbb{R} \backslash \mathbb{Z}$ and $k=1$, however, then it follows from Theorem 1 that

$$
\sum_{q \geq 1} \frac{\widetilde{c}_{q}(x)}{q^{2}}=\sum_{1 \leq q<2 \pi|x|} \frac{\widetilde{c}_{q}(x)}{q^{2}}+\frac{e^{2 \pi i x}-1}{2 \pi i x} \sum_{q>2 \pi|x|} \frac{\varphi(q)}{q^{2}}+\sum_{q>2 \pi|x|} \frac{\mathcal{O}_{x}\left(\sigma_{0}(q)\right)}{q^{2}} .
$$

The sum in the first term is obviously finite, and the series in the last term converges absolutely because $\sum_{q \geq 1} \frac{\sigma_{0}(q)}{q^{2}}=\zeta(2)^{2}$. However, the series in the middle term diverges because

$$
\sum_{q>2 \pi|x|} \frac{\varphi(q)}{q^{2}}+\mathcal{O}_{x}(1)=\sum_{q \geq 1} \frac{\varphi(q)}{q^{2}}=\lim _{k \rightarrow 1^{+}} \frac{\zeta(k)}{\zeta(k+1)} .
$$

Therefore, $\widetilde{\sigma}_{1}(x)$ diverges for all $x \in \mathbb{R} \backslash \mathbb{Z}$.

This behavior is surprising. Although extraordinarily well-behaved on integers, any nonintegral $\epsilon>0$ addition to an integer argument causes $\widetilde{\sigma}_{1}(x)$ to diverge (albeit only logarithmically, as our proof of Corollary 2 shows). Indeed, this is plainly true for any function $\widetilde{f}(x)$ that has $\widehat{f}(q)=\Omega\left(q^{-2}\right)$ in its generalized Ramanujan expansion (5) (assuming it exists). This includes, for example, the generalizations of both Ramanujan's and Hardy's expansions of the zero function [2, 5],

$$
\widetilde{0}_{\operatorname{Ram}}(x):=\sum_{q \geq 1} \frac{\widetilde{c}_{q}(x)}{q} \text { and } \widetilde{0}_{\mathrm{Har}}(x):=\sum_{q \geq 1} \frac{\widetilde{c}_{q}(x)}{\varphi(q)},
$$

as well as the generalization of the number-of-divisors function [5],

$$
\widetilde{\sigma}_{0}(x):=-\sum_{q \geq 1} \frac{\log q}{q} \widetilde{c}_{q}(x)
$$

It is evident from Theorem 1 that these series converge if and only if $x \in \mathbb{Z}$.

For $\sigma_{0}(n)$ and $\sigma_{1}(n)$, we are able to construct continuous and absolutely convergent Ramanujan expansions that interpolate these functions, although only locally on $\mathbb{R}$. We describe these constructions in the next section.

\section{Real Variable Ramanujan Expansions of $\sigma_{0}(n)$ And $\sigma_{1}(n)$}

Fix $\alpha \in \mathbb{Z} \backslash\{0\}$ and denote its divisors by $d_{1}, \ldots, d_{N}$, where $N=\sigma_{0}(\alpha)$. Define $P_{\alpha}(x)$ as the degree- $N$ polynomial whose roots are $d_{1}, \ldots, d_{N}$ :

$$
P_{\alpha}(x):=\prod_{i=1}^{N}\left(x-d_{i}\right)=\sum_{n=0}^{N} a_{n} x^{n} .
$$


The polynomial coefficients $a_{n}$ are given by

$$
a_{n}=(-1)^{N-n} e_{N-n}\left(d_{1}, \ldots, d_{N}\right),
$$

where $e_{j}$ is the $j$ th elementary symmetric polynomial in the divisors of $\alpha$ :

$$
e_{j}\left(d_{1}, \ldots, d_{N}\right):=\sum_{1 \leq i_{1}<\cdots<i_{j} \leq N} d_{i_{1}} \cdots d_{i_{j}} .
$$

Since $P_{\alpha}(d)=0$ for each $d \in\left\{d_{1}, \ldots, d_{N}\right\}$, we have the linear system of equations

$$
\left\{\begin{array}{c}
P_{\alpha}\left(d_{1}\right)=a_{N} d_{1}^{N}+\cdots+a_{1} d_{1}+a_{0}=0 \\
P_{\alpha}\left(d_{2}\right)=a_{N} d_{2}^{N}+\cdots+a_{1} d_{2}+a_{0}=0 \\
\vdots \\
P_{\alpha}\left(d_{N}\right)=a_{N} d_{N}^{N}+\cdots+a_{1} d_{N}+a_{0}=0
\end{array}\right.
$$

In fact, we have for any natural number $k$ the more general system

$$
\left\{\begin{array}{c}
a_{N} d_{1}^{k+N}+\cdots+a_{1} d_{1}^{k+1}+a_{0} d_{1}^{k}=0 \\
a_{N} d_{2}^{k+N}+\cdots+a_{1} d_{2}^{k+1}+a_{0} d_{2}^{k}=0 \\
\vdots \\
a_{N} d_{N}^{k+N}+\cdots+a_{1} d_{N}^{k+1}+a_{0} d_{N}^{k}=0 .
\end{array}\right.
$$

Adding the equations in this second system implies

$$
a_{N}\left(d_{1}^{k+N}+\cdots+d_{N}^{k+N}\right)+\cdots+a_{0}\left(d_{1}^{k}+\cdots+d_{N}^{k}\right)=0
$$

which is equivalent to

$$
a_{N} \sigma_{k+N}(\alpha)+\cdots+a_{0} \sigma_{k}(\alpha)=0 .
$$

By (12) and the fact that no divisor of $\alpha$ is zero, $a_{0}=d_{1} \cdots d_{N} \neq 0$. Consequently,

$$
\sigma_{k}(\alpha)=-\frac{1}{a_{0}}\left(a_{N} \sigma_{k+N}(\alpha)+\cdots+a_{1} \sigma_{k+1}(\alpha)\right) .
$$

Now, on substituting equations (31) and (12) into (13), we obtain a new and nontrivial formula for the divisor function $(k \geq 0, \alpha \in \mathbb{Z} \backslash\{0\})$ :

$$
\sigma_{k}(\alpha)=\sum_{q \geq 1} \sum_{j=0}^{N-1}\left[\frac{(-1)^{j+1} e_{j}\left(d_{1}, \ldots, d_{N}\right)}{d_{1} \cdots d_{N}} \cdot \frac{\zeta(N+k+1-j) \alpha^{N+k-j}}{q^{N+k+1-j}}\right] c_{q}(\alpha) .
$$

This equation constitutes a Ramanujan expansion of $\sigma_{k}(n)$, albeit an "impure" one as the Ramanujan coefficients depend on the argument $\alpha$.

We now take $k=1$ in (13) , and redefine $\widetilde{\sigma}_{1}(x)$ in (6) by the continuous generalization of (13) $: 2$

$$
\widetilde{\sigma}_{1}(x, \alpha):=-\frac{1}{a_{0}}\left(a_{N} \widetilde{\sigma}_{N+1}(x)+\cdots+a_{1} \widetilde{\sigma}_{2}(x)\right) .
$$

Corollary 2 proves that the $\widetilde{\sigma}_{i+1}(x)$ functions on the right side converge absolutely for all $x \in \mathbb{R}$ because $i>0$. It follows that $\widetilde{\sigma}_{1}(x, \alpha)$ converges absolutely for all $x \in \mathbb{R}$. On substituting equations (9) and (12) into (15), we obtain the continuous analogue of (14) with $k=1$ :

$$
\widetilde{\sigma}_{1}(x, \alpha)=\sum_{q \geq 1} \sum_{j=0}^{N-1}\left[\frac{(-1)^{j+1} e_{j}\left(d_{1}, \ldots, d_{N}\right)}{d_{1} \cdots d_{N}} \cdot \frac{\zeta(N+2-j) x^{N+1-j}}{q^{N+2-j}}\right] \widetilde{c}_{q}(x) .
$$

\footnotetext{
${ }^{2}$ We explicitly include in $\widetilde{\sigma}_{1}(x, \alpha)$ a functional dependence on $\alpha$ to highlight the fact that $\alpha$ is implicit in the $a_{n}$ coefficients.
} 
Unlike (6), this constitutes a continuous and absolutely convergent Ramanujan expansion for $\widetilde{\sigma}_{1}(x, \alpha)$.

By a similar construction with $k=0$ in (13), but in which we replace $\sigma_{1}(\alpha)$ by (16), we redefine $\widetilde{\sigma}_{0}(x)$ in (10) to

$$
\widetilde{\sigma}_{0}(x, \alpha):=-\frac{1}{a_{0}}\left(a_{N} \widetilde{\sigma}_{N}(x)+\cdots+a_{2} \widetilde{\sigma}_{2}(x)+a_{1} \widetilde{\sigma}_{1}(x, \alpha)\right) .
$$

On inserting equations (9) and (12) into (17), we obtain an absolutely convergent and continuous Ramanujan expansion for $\widetilde{\sigma}_{0}(x, \alpha)$, akin to $\widetilde{\sigma}_{1}(x, \alpha)$ in (16).

On what subset of $\mathbb{R}$ do (16) and (17) correctly interpolate $\sigma_{1}(n)$ and $\sigma_{0}(n)$, respectively? The answer, unfortunately, is on a local subset only - namely, the open neighborhood $(\alpha-1, \alpha+1) \subset$ $\mathbb{R}$. This follows because (13) presupposes a fixed integer $\alpha$ by which the coefficients in (13) are defined. It is plain, then, that only in a neighborhood of $\alpha$ containing no other integers will the equality (13) necessarily hold, since the polynomial coefficients in (11) for any integer other than $\alpha$ are generally different from those in $P_{\alpha}(x)$.

So while we have shown that there exist continuous and absolutely convergent Ramanujan expansions that interpolate $\sigma_{0}(n)$ and $\sigma_{1}(n)$, they do so only locally in the neighborhood around a given integer. This begs the question of whether there exists a continuous and absolutely convergent Ramanujan expansion that interpolates $\sigma_{1}(n)$ (and perhaps even $\sigma_{0}(n)$ ) globally on $\mathbb{R}$, or at least on the positive reals.

\section{REFERENCES}

1. E. Cohen, Arithmetical functions associated with the unitary divisors of an integer, Math. Z. 74 (1960), 66-80.

2. G. H. Hardy, Ramanujan: Twelve lectures on subjects suggested by his life and work, Cambridge University Press, Cambridge, 1940.

3. L. G. Lucht, A survey of Ramanujan expansions, Int. J. Number Theory 6 (2010), 1785-1799.

4. M. R. Murty, Ramanujan series for arithmetical functions, Hardy-Ramanujan J. 36 (2013), 21-33.

5. S. Ramanujan, On certain trigonometric sums and their applications in the theory of numbers, Transactions of the Cambridge Phil. Society 22 (1918), 179-199.

6. W. Schwarz and J. Spilker, Arithmetical functions, London Mathematical Society Lecture Note Series, vol. 184, Cambridge University Press, Cambridge, 1994.

7. R. Sivaramakrishnan, Classical theory of arithmetic functions, Monographs and Textbooks in Pure and Applied Mathematics, vol. 126, Marcel Dekker, Inc., New York, 1989.

8. L. Tóth, Ramanujan expansions of arithmetic functions of several variables, Ramanujan J. 47 (2018), 589-603.

9. N. Ushiroya, Ramanujan-Fourier series of certain arithmetic functions of two variables, Hardy-Ramanujan J. 39 (2016), 1-20.

Department of Physics, Harvey Mudd College

Email address: msfox@hmc.edu

Department of Mathematics, Harvey Mudd College

Email address: ckaramchedu@hmc.edu 